\title{
Reference-Free Displacement Estimation of Bridges Using Kalman Filter-Based Multimetric Data Fusion
}

\author{
Soojin Cho, ${ }^{1}$ Jong-Woong Park, ${ }^{2}$ Rajendra P. Palanisamy, ${ }^{3}$ and Sung-Han Sim ${ }^{3}$ \\ ${ }^{1}$ Department of Civil Engineering, University of Seoul, Seoul 02504, Republic of Korea \\ ${ }^{2}$ Department of Civil and Environmental Engineering, University of Illinois at Urbana-Champaign, Urbana, IL 61801, USA \\ ${ }^{3}$ School of Urban and Environmental Engineering, Ulsan National Institute of Science and Technology (UNIST), \\ Ulsan 44919, Republic of Korea
}

Correspondence should be addressed to Sung-Han Sim; ssim@unist.ac.kr

Received 18 February 2016; Revised 5 July 2016; Accepted 17 August 2016

Academic Editor: Marco Consales

Copyright (C) 2016 Soojin Cho et al. This is an open access article distributed under the Creative Commons Attribution License, which permits unrestricted use, distribution, and reproduction in any medium, provided the original work is properly cited.

\begin{abstract}
Displacement responses of a bridge as a result of external loadings provide crucial information regarding structural integrity and current conditions. Due to the relative characteristic of displacement, the conventional measurement approach requires reference points to firmly install the transducers, while the points are often unavailable for bridges. In this paper, a displacement estimation approach using Kalman filter-based data fusion is proposed to provide a practical means for displacement measurement. The proposed method enables accurate displacement estimation by optimally utilizing acceleration and strain in combination that have high availability and are free from reference points for sensor installation. The Kalman filter is formulated using a state-space model representing the double integration of acceleration and model-based strain-displacement relationship. The validation of the proposed method is conducted successfully by a numerical simulation and a field experiment, which shows the efficacy and accuracy of the proposed approach in bridge displacement measurement.
\end{abstract}

\section{Introduction}

Structural health monitoring (SHM) has been considered as an essential procedure for the sustainability of bridges which are the national assets supporting reliable and efficient operation of our society. Technology advances in SHM research have expanded beyond theoretical development and laboratory-scale verification to real-world applications [1-3]. SHM is generally composed of three processes: (1) acquisition of structural responses and environmental factors, (2) assessment of structural condition based on the measurement, and (3) maintenance action when required based on the assessment. Because the first task builds the foundation of the whole SHM procedure, data acquisition is a key component for successful SHM. Before acquiring the structural responses, the types of physical quantities to be monitored are determined priorly with considering necessity and availability of responses. The availability generally includes the accuracy, accessibility, convenience, and cost in the measurement.
Acceleration and strain are the most widely measured responses for the SHM due to their high availability. Acceleration is generally sensitive in measuring dynamic characteristics of a large structure with high convenience in the installation, and strain can be directly converted into current stress condition resisted by the instrumented structural member. Though acceleration and strain possess the majority of the measured responses for the SHM, other responses are often required in practice with specific purposes, including displacement [4-6], inclination [7], structural impedance [8], and $\mathrm{pH}[9]$.

Displacement is an intuitive response generated by external forces applied to a structure. Displacement has been used as a practical measurement that indicates structural stability and soundness. Indeed, a variety of modern design codes adopt limit displacement levels under given loadings to assure structural safety. For example, Korea and the US limit maximum deflections of vehicular bridges up to $1 / 800$ of the bridge span length $[10,11]$ and Canada uses the maximum deflection and the first natural frequency for the evaluation of 
pedestrian bridges [12]. The practical usage of displacement in the design has been extended to various efforts to use it in the SHM of full-scale civil engineering structures [13-15].

Despite the intuitional characteristic and high demands in practice, displacement has not been as popular as acceleration and strain. Displacement is a relative measurement whose transducer must be placed between a measurement point and a stationary reference point. The sensor installation on bridges is often challenging because bridges are generally constructed over a road, a river, a swamp, or a sea, where a proper reference point is difficult to find. Though a number of noncontact transducers have been developed to resolve the issue, such as global positioning system (GPS) [1618], LASER-based transducer $[19,20]$, and computer vision system [21-24], they are still unpopular due to the high cost and synchronization issues when densely deployed.

A noticeable alternative is indirect estimation approaches that convert other structural responses to displacement based on their physical relationships. The convertible responses include acceleration [25-27], strain [28-31], and inclination [32]. Knowing the fact that these responses can be measured without the reference point, the indirect estimation approaches have quite strong potential to be widely used in field applications. However, the indirect methods generally suffer from the low accuracy due to numerical errors and/or imperfect physical relationships. For example, the conversion of acceleration to displacement involves large low-frequency drift errors occurring in a numerical integration [25]; the conversion of strain exhibits errors due to an assumed mapping model between strain and displacement [31]. Thus, the indirect estimation approach has not been commonly accepted in the SHM field yet.

The accuracy enhancement can be achieved by employing multimetric (i.e., a heterogeneous mixture of multiple responses) data based on the data fusion model, as recently reported in [33-36]. The synergic usage of multimetric data minimizes the drawback of each sole response as observed in the indirect displacement estimation methods in the literature. Roberts et al. reported that the conversion of acceleration could be enhanced by referencing displacement measured by a high-precision GPS in the Kalman filtering [37]. Another effort for employing multimetric data is a data fusion method that uses acceleration and strain in combination [38-42]. The method has showed significantly improved accuracy, though a low-frequency noise in strain measurement still brings undesired errors in the estimation. In the aerospace engineering field, the Kalman filterbased data fusion has been actively studied for the accuracy enhancement of vehicle attitude and position assessment [43, 44], autonomous navigation of unmanned vehicles [45, 46], and indoor positioning $[47,48]$. Many of them are available on the market by the company such as Trimble Navigation Limited [48] and SBG Systems [49].

In this paper, a Kalman filter-based indirect estimation method is proposed for convenient but accurate measurement of displacement on bridges. The proposed method uses both acceleration and strain, which have the highest availability on bridges without the reference point issue. Furthermore, acceleration and strain have sensitivity in relatively high- and low-frequency range, respectively; the fusion of acceleration and strain accurately estimates displacement in a broad frequency region by taking the synergic effect. The Kalman filter has been selected as the data fusion model due to its high reliability and versatility. A mathematical formulation of the Kalman filter for the displacement estimation is presented based on the double integration of acceleration and modalmapping of strain. The performance and efficacy of the proposed method are validated in a numerical simulation using a simply supported beam model and further investigated in a field experiment on a single-span prestressed concrete bridge.

\section{Formulation for Displacement Estimation Using Kalman Filter}

This section provides a mathematical formulation for the proposed displacement estimation approach using the Kalman filter to use acceleration and strain measurements in combination. The fundamental idea of the proposed method is to optimally extract necessary information for displacement estimation from two different measurements, which the sole use of each measurement is not able to do. In general, acceleration responses have rich information in the high-frequency region, whereas strains are sensitive in the low-frequency and static regions. The numerical double integration of an acceleration measurement yields the corresponding displacement at the same position with accurate dynamic components, while resulting in the large low-frequency drift. This error can be compensated by introducing the strain-displacement relationship, which generally does not involve the numerical integration. Thus, selectively utilizing information contained in each measurement can lead to accurate displacement estimation in all frequency regions.

The Kalman filter for displacement estimation needs to be carefully designed to enable such optimal selective filtering. In this study, the formulation in [33] to fuse acceleration and displacement sampled at different rates is modified to accommodate acceleration and strain measurements. The statespace model for the acceleration-displacement relationship can be written using the definition of acceleration, which is the second derivative of displacement with respect to time, as

$$
\begin{aligned}
{\left[\begin{array}{l}
\dot{x}(t) \\
\ddot{x}(t)
\end{array}\right] } & =\left[\begin{array}{ll}
0 & 1 \\
0 & 0
\end{array}\right]\left[\begin{array}{l}
x(t) \\
\dot{x}(t)
\end{array}\right]+\left[\begin{array}{l}
0 \\
1
\end{array}\right] a(t)+\left[\begin{array}{l}
0 \\
1
\end{array}\right] w(t), \\
u(t) & =\left[\begin{array}{ll}
1 & 0
\end{array}\right]\left[\begin{array}{l}
x(t) \\
\dot{x}(t)
\end{array}\right]+v(t),
\end{aligned}
$$

where $x(t), a(t), u(t), w(t)$, and $v(t)$ are state variable, acceleration, displacement, and measurement noises associated with acceleration and displacement, respectively. $w(t)$ and $v(t)$ are random processes with the Gaussian distribution with covariance of $q$ and $r$. Because all measured signals are 
discrete, a state-space model in the discrete-time domain is desired. The discrete version of (1) can be written as

$$
\begin{aligned}
x_{k} & =\left[\begin{array}{cc}
1 & d t \\
0 & 1
\end{array}\right] x_{k-1}+\left[\begin{array}{c}
\frac{d t^{2}}{2} \\
d t
\end{array}\right] a_{k}+w_{k-1} \\
& =A x_{k-1}+B a_{k}+w_{k-1}, \\
u_{k} & =\left[\begin{array}{ll}
1 & 0
\end{array}\right] x_{k}+v_{k}=H x_{k}+v_{k},
\end{aligned}
$$

where $a_{k}, u_{k}$ are the measured acceleration and displacement, respectively, and $w_{k}$ and $v_{k}$ are the corresponding noise processes. The noise processes have the covariance matrices defined as

$$
\begin{aligned}
& Q=q\left[\begin{array}{cc}
\frac{d t^{3}}{3} & \frac{d t^{2}}{2} \\
\frac{d t^{2}}{2} & d t
\end{array}\right] \\
& R=\frac{r}{d t}
\end{aligned}
$$

where $d t$ is the sampling time.

The discrete-time state-space model in (2) and (3) is then utilized to develop the Kalman filter for displacement estimation. The measured acceleration is used to project the state ahead via the transition matrix $A$ as

$$
\begin{aligned}
& \widehat{x}_{k}^{-}=A \widehat{x}_{k-1}+B a_{k}, \\
& P_{k}^{-}=A P_{k-1} A^{\mathrm{T}}+Q,
\end{aligned}
$$

where $\widehat{x}_{k}^{-}$and $\widehat{x}_{k}$ are respective prior and updated state estimates and $P_{k}^{-}$and $P_{k}$ are respective prior and updated error covariance matrices. Subsequently, the Kalman gain $K_{k}$ is computed as

$$
K_{k}=P_{k}^{-} H^{\mathrm{T}}\left(H P_{k}^{-} H^{\mathrm{T}}+R\right)^{-1} .
$$

The measured strain is then employed to update the estimate with the Kalman gain obtained in (5):

$$
\begin{aligned}
& \widehat{x}_{k}=\widehat{x}_{k}^{-}+K_{k}\left(\alpha u_{k}-H \widehat{x}_{k}^{-}\right), \\
& P_{k}=\left(1-K_{k} H\right) P_{k}^{-},
\end{aligned}
$$

where $u_{k}$ is the displacement converted from measured strain data and $\alpha$ is an error correction factor for $u_{k}$ [38]. The updated state yields the estimated displacement as

$$
\widehat{u}_{k}=\left[\begin{array}{ll}
1 & 0
\end{array}\right] \widehat{x}_{k} .
$$

This Kalman filter formulation for the displacement estimation is based on the state-space model that describes the definition of acceleration in the time updated in (4) and the strain-displacement relationship in the measurement update in (6). While the acceleration definition as the second derivative of displacement is clearly seen in (4), the straindisplacement relationship needs to be determined depending on structures and measurement locations.
The conversion relationship from strain to displacement can be obtained from a numerical model of a structure [2831]. Displacement and strain responses can be written in the modal coordinates as

$$
\begin{aligned}
& \underset{u}{u}(t)=\sum_{i} \phi_{i} q_{i}(t)=\Phi \underset{\sim}{q}(t) \\
& \underset{\sim}{\varepsilon}(t)=\sum_{i} \psi_{i} q_{i}(t)=\underline{\Psi}_{\underline{q}}(t),
\end{aligned}
$$

where $\underset{\sim}{\mathcal{u}}$ and $\underset{\sim}{\varepsilon}$ are respective displacement and strain vectors; $\phi_{i}$ and $\psi_{i}$ are respective displacement mode shapes of displacement and strain in the $i$ th mode; $\Phi$ and $\Psi$ are respective mode shape matrices of displacement and strain; $q_{i}$ is the $i$ th modal coordinate; and $\underset{\sim}{q}$ is the modal coordinate vector. The conversion relationship is obtained as

$$
\underline{u}=\Phi \Psi^{\dagger} \underline{\mathcal{\varepsilon}}
$$

where $\dagger$ denotes the matrix pseudoinverse. Given a finite element model of a bridge, the conversion relationship in (9) is readily available. One particular and common type of bridges is a single-span bridge with homogeneous sectional properties and simply supported boundary conditions. The mode shapes can be reasonably assumed to be sinusoidal functions, which allow (9) to be written as [31]

$$
\underset{\sim}{u}=\frac{L^{2}}{y \pi^{2}} \Phi\left[\begin{array}{ccc}
\sin \frac{\pi z_{1}}{L} & \cdots & r^{2} \sin \frac{r \pi z_{1}}{L} \\
\vdots & \ddots & \vdots \\
\sin \frac{\pi z_{n}}{L} & \cdots & r^{2} \sin \frac{r \pi z_{n}}{L}
\end{array}\right]^{\dagger} \underset{\sim}{\mathcal{E}}
$$

where $y$ is the location of the neutral axis, $r$ is the maximum number of natural modes being used, $z_{1}, \ldots, z_{n}$ are the locations of strain measurements, and $L$ is the beam length. To compensate the inevitable discrepancy between physical structures and the numerical model such as the finite element model and the simple-beam model, the error correction factor $\alpha$ is employed. More detailed information regarding $\alpha$ can be found in [38].

The proposed displacement estimation method has a distinct advantage in accurate displacement estimation in both low- and high-frequency regions with a minimized noise floor. The displacement contained in the state estimate in the time update of (4) inherits the frequency content of the measured acceleration, which has rich information in the high-frequency region. Estimated from the acceleration, the displacement has a low-frequency noise floor, whereas the large low-frequency drift is involved. The measurement update in (6) is to compensate this low-frequency drift error by employing the strain-displacement relationship. Thus, the resulting displacement of the Kalman filter shall be accurate in all frequency regions. In the following numerical example, the performance of the proposed approach will be verified regarding these aspects of accuracy in both time and frequency domains. 




Figure 1: Beam model.

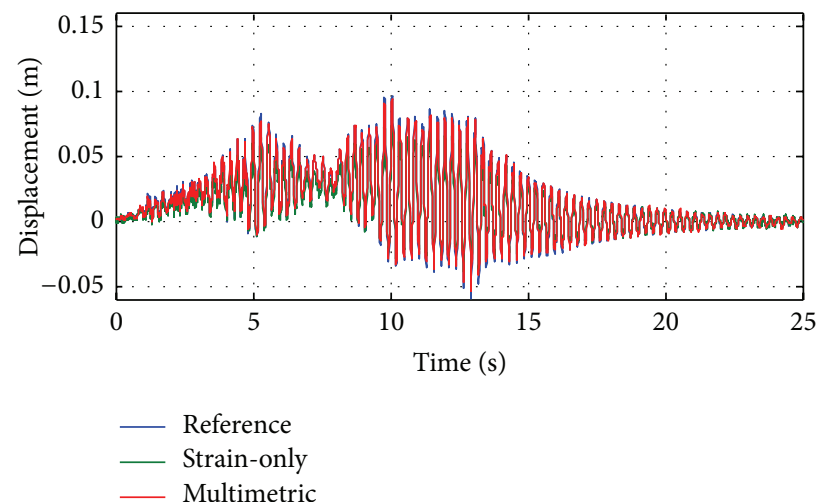

(a)

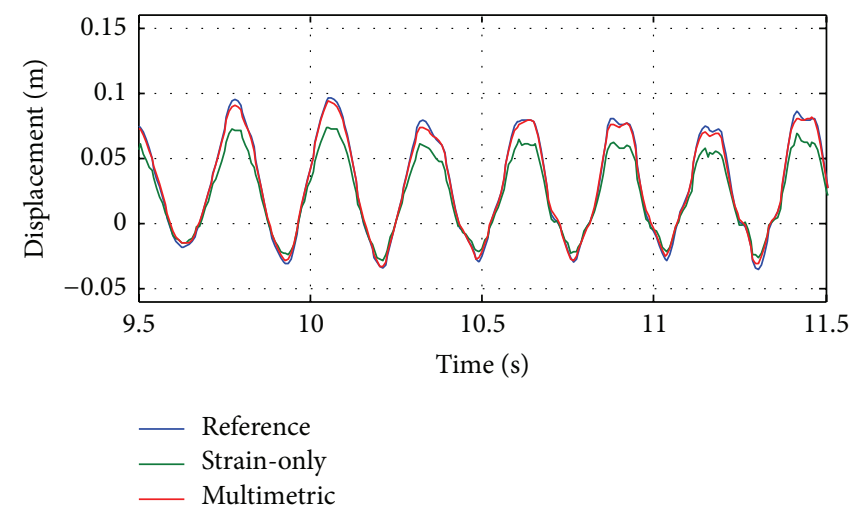

(b)

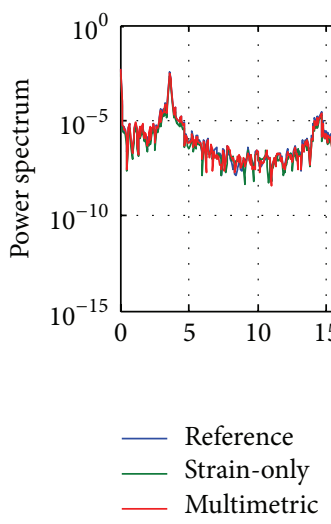

(c)

FIGURE 2: Estimated and reference displacement at node 5: (a) time domain data, (b) close-up view of (a), and (c) frequency domain data.

TABLE 1: Beam properties.

\begin{tabular}{lc}
\hline Properties & Values \\
\hline Length & $1.6 \mathrm{~m}$ \\
Thickness & $4 \mathrm{~cm}$ \\
Width & $15 \mathrm{~cm}$ \\
Elastic modulus & $206 \mathrm{GPa}$ \\
Mass density & $7850 \mathrm{~kg} / \mathrm{m}^{3}$ \\
\hline
\end{tabular}

\section{Numerical Validation}

To validate the proposed displacement estimation method, a beam model with a moving load shown in Figure 1 is considered. The beam is modeled as simply supported with 16 Euler beam elements, and its property is tabulated in Table 1. The moving load is introduced to simulate truck loading which is often used in bridge testing. The load moving at the speed of $0.1 \mathrm{~m} / \mathrm{s}$ is designed to have a dynamic variance in time using a normal distribution (mean: $10 \mathrm{~N}$, standard deviation: $3 \mathrm{~N}$ ). Time histories of strain and acceleration responses from the beam under the moving load are simulated at nodes 5, 9, and 13 shown in Figure 1. 5\% and $10 \%$ RMS noise signals are introduced to acceleration and strain, respectively. Displacement responses at the nodes are also directly calculated from the beam model to verify the accuracy of the estimation using acceleration and strain data.

The performance of the proposed method is evaluated by comparing the estimated displacements with those obtained by the strain-only method using the strain-displacement relationship [28, 31] and the reference displacements. As the simply supported beam is considered in this numerical example, (10) is utilized to obtain the strain-displacement relationship for the strain-only and the proposed methods. A model error is introduced by intentionally using $2.6 \mathrm{~cm}$ as the location of the neutral axis (i.e., $y$ of (10)), of which the true location is $2 \mathrm{~cm}$. As previously described, the error correction factor $\alpha$ to compensate this model error [38] is used in the estimation process.

The comparison for displacements at nodes 5 and 9 is shown in Figures 2 and 3, respectively. Figures 2(a) and 3(a) 


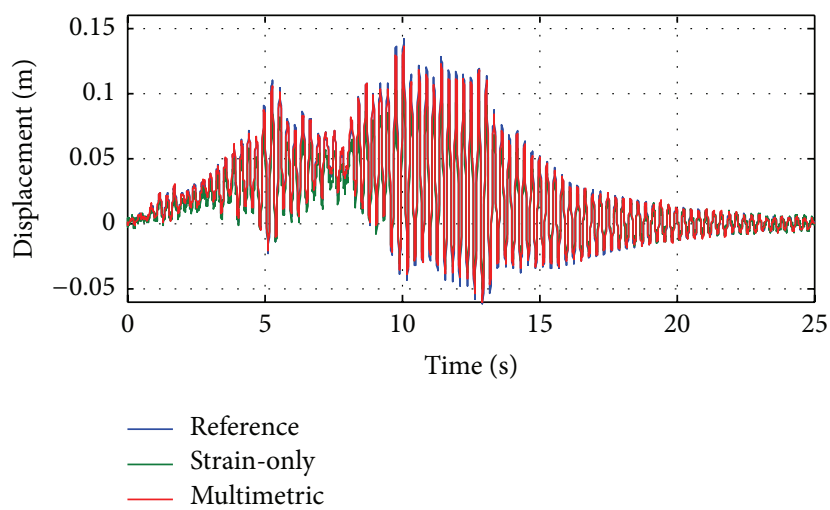

(a)

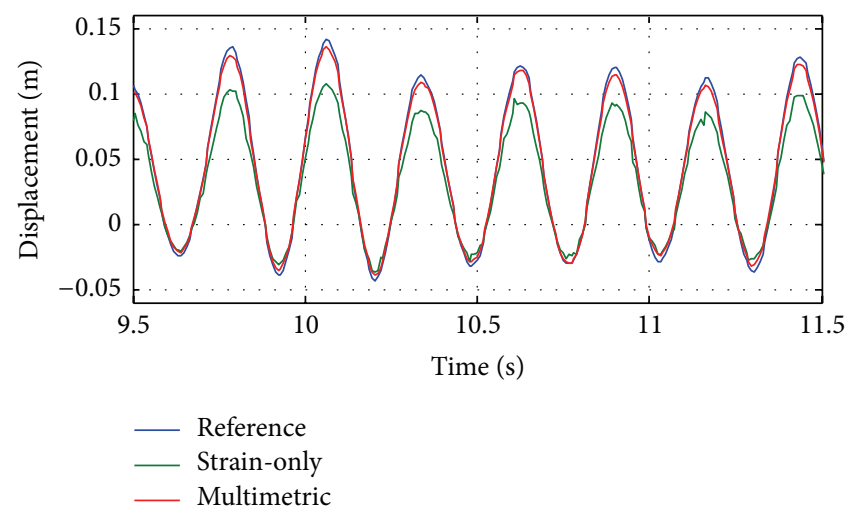

(b)

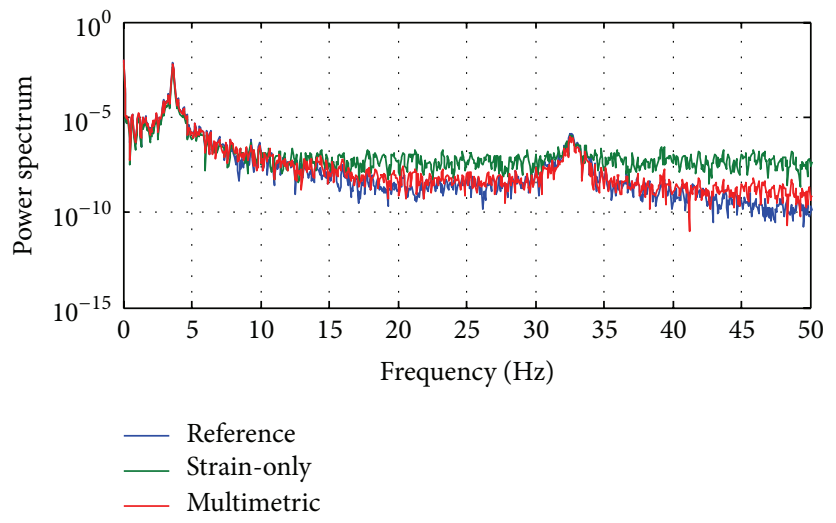

(c)

FIgURE 3: Estimated and reference displacement at node 9: (a) time domain data, (b) close-up view of (a), and (c) frequency domain data.

show that the displacements obtained by the strain-only and the proposed method apparently agree well with the reference displacements. However, the close-up views (i.e., Figures 2(b) and $3(b))$ and the frequency domain data obtained (i.e., Figures 2(c) and 3(c)) obviously show that the proposed method exhibits better performance in both the low- and highfrequency regions. The close-up views (i.e., Figures 2(b) and 3(b)) from 9.5 to 11.5 seconds show that the proposed method estimates the amplitude of the displacement (related to lowfrequency accuracy) more accurately than the strain-based method. This is because of the intentional introduction of erroneous neutral axis to simulate the possible model error to develop the strain-displacement relationship. In the proposed method, the error correction factor $\alpha$, which is only available when both acceleration and strain are measured together, is used to calibrate the amplitude of displacement, whereas the sole strain measurement does not allow calculating $\alpha$. Meanwhile, the frequency domain data obtained by fast Fourier transform (i.e., Figures 2(c) and 3(c)) shows that the noise floor (related to high-frequency accuracy) of the proposed method is lower than that of the strain-only method.

The quantitative estimation error, defined in terms of $u_{r}$ and $u_{\varepsilon}$ as (11), is calculated for the obtained displacements shown in Figures 2 and 3:

$$
E_{\mathrm{err}}(\%)=\frac{\sigma\left(u_{r}-u_{\varepsilon}\right)}{\sigma\left(u_{r}\right)} \times 100
$$

The estimation errors $E_{\text {err }}$ of the proposed method are $7.4 \%$ and $6.0 \%$ for the displacements at nodes 5 and 9 , respectively. These error levels can be considered to be reasonably low and agree with the graphical observations from Figures 2 and 3 .

\section{Experimental Validation on a Test Bridge}

The experimental validation of the proposed method is carried out on the prestressed concrete bridge shown in Figure 4 . The bridge has a single span with four prestressed concrete girders, and its span length is $11 \mathrm{~m}$. A truck of 28.93 ton is used to load the bridge. The truck runs on the bridge with two different speeds: (1) $5 \mathrm{~km} / \mathrm{h}$ and (2) $15 \mathrm{~km} / \mathrm{h}$. The bridge is instrumented to measure the reference displacement as well as acceleration and strain for the proposed method. The reference displacements are measured by two laser displacement sensors at Locations B and C marked in Figure 4 . For the proposed method, three strain gauges are installed at Locations A, C, and D and two accelerometers are placed at the same locations where the displacements are to be estimated and compared with the reference displacements (i.e., Locations B and C).

The displacements at Locations B and C are estimated by the proposed method. The neutral axis of the bridge is assumed to be $0.25 \mathrm{~m}$ for (10), whose error is to be compensated by the calibration factor $\alpha$ as described in Section 2. The displacements estimated at two locations 


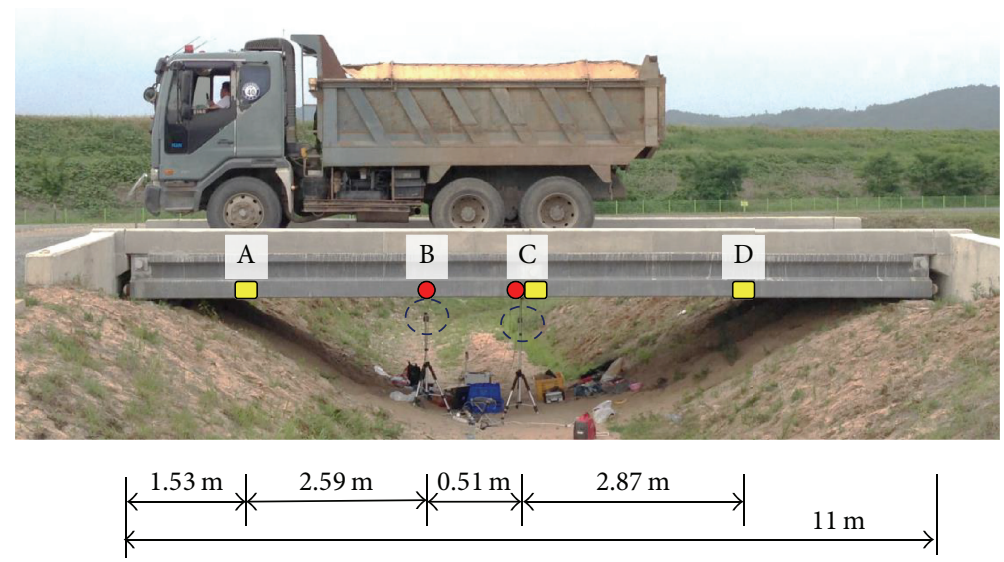

$\square$ Strain gage
Accelerometer
- Laser displacement sensor

FIGURE 4: Prestressed concrete bridge under the truck loading and sensor configuration.

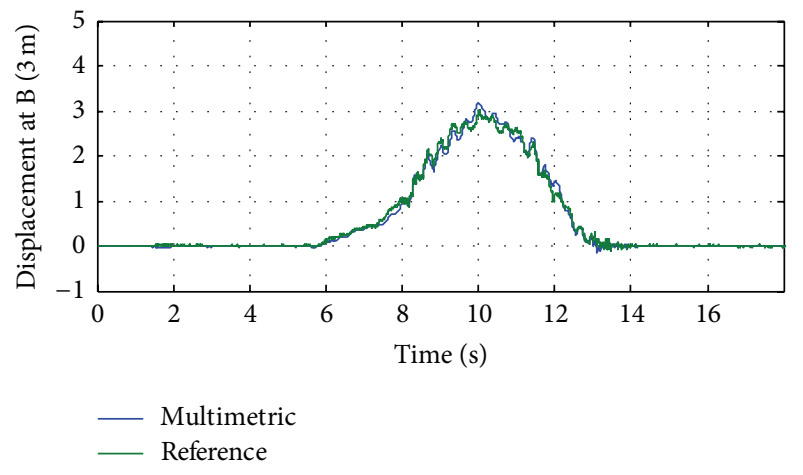

(a)

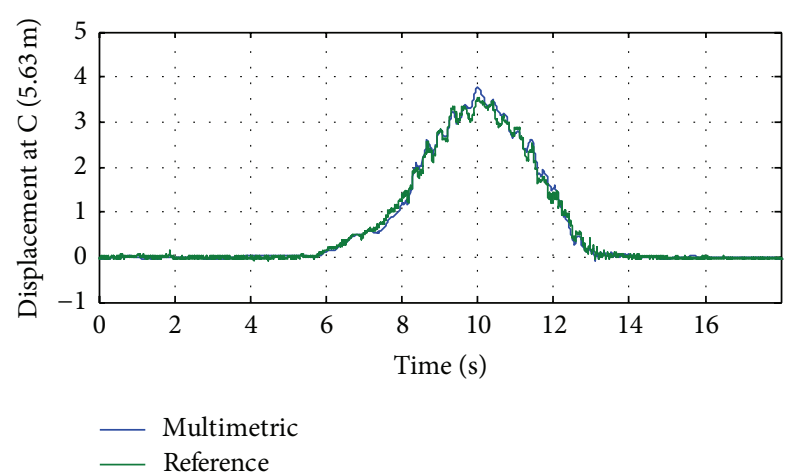

(b)

FIGURE 5: Comparison of estimated and reference displacements (truck speed: $5 \mathrm{~km} / \mathrm{h}$ ): (a) displacements at Location B and (b) displacements at Location $\mathrm{C}$.

by the proposed method are compared with the references measured by the laser displacement sensors in Figures 5 and 6 for both truck speeds. The estimated displacements show a good correlation with the reference displacements for both locations and both truck speeds. The errors are quantified between 7.6 and $8.3 \%$ by (11) as tabulated in Table 2. As to the location, the error is smaller at the center of the bridge (i.e., Location C), which seems substantial considering larger amplitude of the displacement at Location C. The errors are not seriously affected by the different truck speeds, which may infer different loading patterns. The errors are slightly larger than those obtained in the numerical simulation (i.e., $7.4 \%$ and $6.0 \%$ ). Considering that the experiment is carried out on a large structure, the errors evidently show the good performance of the proposed method. Therefore, the proposed method can obtain structural displacement with high accuracy using highly available responses (i.e., acceleration and strain), especially at the bridges where the conventional displacement transducers are hard to be instrumented.
TABLE 2: Error measurements at Locations B and C.

\begin{tabular}{lccc}
\hline Truck speed & \multicolumn{3}{c}{$E_{\text {est }}(\%)$} \\
& Location B & Location C \\
\hline $5 \mathrm{~km} / \mathrm{h}$ & 8.33 & 7.85 \\
$15 \mathrm{~km} / \mathrm{h}$ & 8.00 & 7.63 \\
\hline
\end{tabular}

\section{Conclusions}

In this paper, a Kalman filter-based approach using multimetric responses (i.e., acceleration and strain) that have reference-free nature was proposed for indirect displacement estimation of bridges. The formulation of the Kalman filter was constructed using a state-space model representing the double integration of acceleration and model-based strain-displacement relationship. The validation was carried out successfully by a numerical simulation and a field 


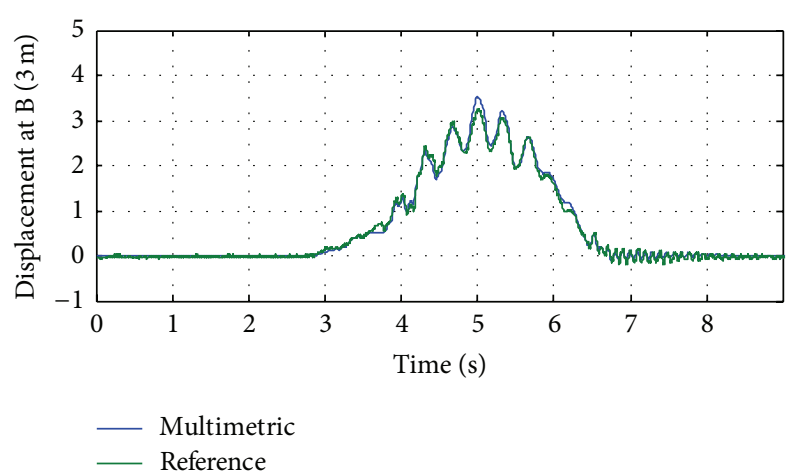

(a)

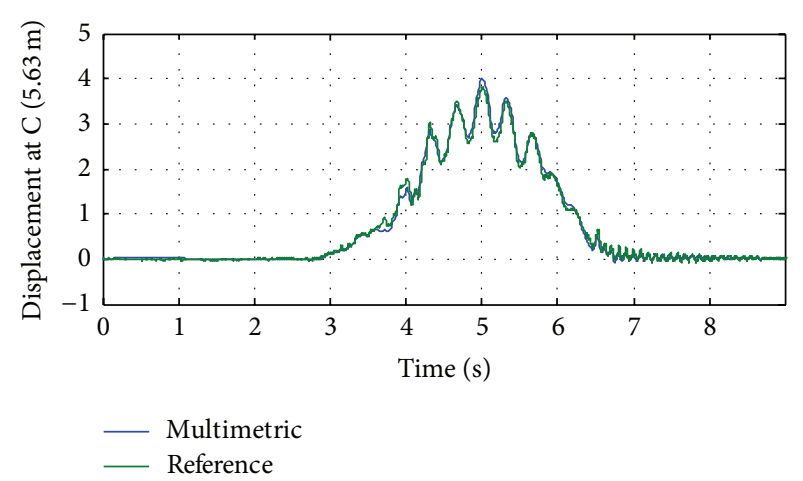

(b)

Figure 6: Comparison of estimated and reference displacements (truck speed: $15 \mathrm{~km} / \mathrm{h}$ ): (a) displacements at Location B and (b) displacements at Location C.

experiment. The results of the validation can be summarized as follows:

(1) In the numerical simulation, the proposed method estimated the nonzero-mean displacements at two locations with good agreement to the reference displacements in both time and frequency domains. The quantitative estimation errors at two locations are 7.4 and $6.0 \%$.

(2) In the experimental test on the prestressed concrete bridge, the proposed method estimated the displacements excited by a truck with the quantitative estimation error between 7.6 and $8.3 \%$. The slightly larger errors than those from the numerical simulation evidently showed good performance of the proposed method on real full-scale bridges.

(3) The errors were not affected by the truck speeds (representing loading patterns) but amplitude of the displacement.

(4) The proposed method is expected to be advantageous for bridge structures where the reference points to stably locate conventional displacement transducers are unavailable.

\section{Competing Interests}

The authors declare that they have no competing interests.

\section{Acknowledgments}

This research was a part of the project titled "Development of Active-Controlled Tidal Stream Generation Technology" funded by the Ministry of Oceans and Fisheries, Korea (20110171).

\section{References}

[1] H. Wang, T. Tao, A. Li, and Y. Zhang, "Structural health monitoring system for Sutong cable-stayed bridge," Smart Structures and Systems, vol. 18, pp. 317-334, 2016.
[2] C.-C. Chen, W.-H. Wu, C.-Y. Liu, and G. Lai, "Damage detection of a cable-stayed bridge based on the variation of stay cable forces eliminating environmental temperature effects," Smart Structures and Systems, vol. 17, no. 6, pp. 859-880, 2016.

[3] J. Seo, G. Hatfield, and J.-H. Kimn, "Probabilistic structural integrity evaluation of a highway steel bridge under unknown trucks," Journal of Structural Integrity and Maintenance, vol. 1, pp. 65-72, 2016.

[4] G. A. Stephen, J. M. W. Brownjohn, and C. A. Taylor, "Measurements of static and dynamic displacement from visual monitoring of the Humber Bridge," Engineering Structures, vol. 15, no. 3, pp. 197-208, 1993.

[5] S. S. Law, H. S. Ward, G. B. Shi, R. Z. Chen, P. Waldron, and C. Taylor, "Dynamic assessment of bridge load-carrying capacities. I," Journal of Structural Engineering, vol. 121, no. 3, pp. 478-487, 1995.

[6] J.-H. Yi, S. Cho, K.-Y. Koo et al., "Structural performance evaluation of a steel-plate girder bridge using ambient acceleration measurements," Smart Structures and Systems, vol. 3, no. 3, pp. 281-298, 2007.

[7] O. Burdet and J. L. Zanella, "Automatic monitoring of bridges using electronic inclinometers," in Proceedings of the IABSE Congress Report, 16th Congress of IABSE, vol. 16, pp. 1574-1581, Lucerne, Switzerland, 2000.

[8] G. Park, H. H. Cudney, and D. J. Inman, "Impedance-based health monitoring of civil structural components," Journal of Infrastructure Systems, vol. 6, no. 4, pp. 153-160, 2000.

[9] G. S. Duffó and S. B. Farina, "Development of an embeddable sensor to monitor the corrosion process of new and existing reinforced concrete structures," Construction and Building Materials, vol. 23, no. 8, pp. 2746-2751, 2009.

[10] American Association of State Highway and Transportation Officials (AASHTO), Load Resistance and Factor Design, Bridge Design Specifications, American Association of State Highway and Transportation Officials (AASHTO), Washington, DC, USA, 2008.

[11] Ministry of Land, Transport, and Maritime (MLTM) Affairs (2012) Korea highway bridge design code (LRFD), 2012.

[12] Ontario Highway Bridge Design Code, Ontario Ministry of Transportation and Communications, 1st edition, 1978.

[13] B. C. Faulkner, F. Barton, T. T. Baber, and W. T. McKeel, "Determination of bridge response using acceleration data," Tech. 
Rep., Virgina Transportation Research Council, Charlottesville, Va, USA, 1996.

[14] S. Sumitoro, Y. Matsui, M. Kono, T. Okamoto, and K. Fujii, "Long span bridge health monitoring system in Japan," in Health Monitoring and Management of Civil Infrastructure Systems, vol. 4337 of Proceedings of SPIE, pp. 517-524, International Society for Optics and Photonics, Newport Beach, Calif, USA, 2001.

[15] T. Miyata, H. Yamada, H. Katsuchi, and M. Kitagawa, "Fullscale measurement of Akashi-Kaikyo Bridge during typhoon," Journal of Wind Engineering and Industrial Aerodynamics, vol. 90, no. 12-15, pp. 1517-1527, 2002.

[16] Y. Fujino, M. Murata, S. Okano, and M. Takeguchi, "Monitoring system of the Akashi Kaikyo Bridge and displacement measurement using GPS," in Nondestructive Evaluation of Highways, Utilities, and Pipelines IV, vol. 3995 of Proceedings of SPIE, pp. 229-236, International Society for Optics and Photonics, San Diego, Calif, USA, June 2000.

[17] T. Kijewski-Correa and M. Kochly, "Monitoring the windinduced response of tall buildings: GPS performance and the issue of multipath effects," Journal of Wind Engineering and Industrial Aerodynamics, vol. 95, no. 9-11, pp. 1176-1198, 2007.

[18] H. Jo, S.-H. Sim, A. Tatkowski, B. F. Spencer Jr., and M. E. Nelson, "Feasibility of displacement monitoring using low-cost GPS receivers," Structural Control and Health Monitoring, vol. 20, no. 9, pp. 1240-1254, 2013.

[19] H. H. Nassif, M. Gindy, and J. Davis, "Comparison of laser Doppler vibrometer with contact sensors for monitoring bridge deflection and vibration," NDT and E International, vol. 38, no. 3, pp. 213-218, 2005.

[20] R. Bai, W. Ostachowicz, M. Radzienski, and M. Cao, "Vibrational damage detection using fractal surface singularities with noncontact laser measurement," Journal of Vibration and Control, vol. 22, no. 11, pp. 2569-2581, 2016.

[21] P. Olaszek, "Investigation of the dynamic characteristic of bridge structures using a computer vision method," Measurement, vol. 25, no. 3, pp. 227-236, 1999.

[22] X. W. Ye, C. Z. Dong, and T. Liu, "Image-based structural dynamic displacement measurement using different multiobject tracking algorithms," Smart Structures and Systems, vol. 17, no. 6, pp. 935-956, 2016.

[23] A. M. Wahbeh, J. P. Caffrey, and S. F. Masri, "A vision-based approach for the direct measurement of displacements in vibrating systems," Smart Materials and Structures, vol. 12, no. 5, pp. 785-794, 2003.

[24] J. J. Lee and M. Shinozuka, "A vision-based system for remote sensing of bridge displacement," NDT \& E International, vol. 39, no. 5, pp. 425-431, 2006.

[25] K.-T. Park, S.-H. Kim, H.-S. Park, and K.-W. Lee, "The determination of bridge displacement using measured acceleration," Engineering Structures, vol. 27, no. 3, pp. 371-378, 2005.

[26] H. S. Lee, Y. H. Hong, and H. W. Park, "Design of an FIR filter for the displacement reconstruction using measured acceleration in low-frequency dominant structures," International Journal for Numerical Methods in Engineering, vol. 82, no. 4, pp. 403434, 2010 .

[27] J. W. Park, S. H. Sim, and H. J. Jung, "Development of a wireless displacement measurement system using acceleration responses," Sensors, vol. 13, no. 7, pp. 8377-8392, 2013.

[28] L.-H. Kang, D.-K. Kim, and J.-H. Han, "Estimation of dynamic structural displacements using fiber Bragg grating strain sensors," Journal of Sound and Vibration, vol. 305, no. 3, pp. 534542, 2007.
[29] S. Rapp, L.-H. Kang, J.-H. Han, U. C. Mueller, and H. Baier, "Displacement field estimation for a two-dimensional structure using fiber Bragg grating sensors," Smart Materials and Structures, vol. 18, no. 2, Article ID 025006, 2009.

[30] S.-J. Chang and N.-S. Kim, "Estimation of displacement response from FBG strain sensors using empirical mode decomposition technique," Experimental Mechanics, vol. 52, no. 6, pp. 573-589, 2012.

[31] S. Shin, S.-U. Lee, Y. Kim, and N.-S. Kim, "Estimation of bridge displacement responses using FBG sensors and theoretical mode shapes," Structural Engineering and Mechanics, vol. 42, no. 2, pp. 229-245, 2012.

[32] X. Hou, X. Yang, and Q. Huang, "Using inclinometers to measure bridge deflection," Journal of Bridge Engineering, vol. 10, no. 5, pp. 564-569, 2005.

[33] A. Smyth and M. Wu, "Multi-rate Kalman filtering for the data fusion of displacement and acceleration response measurements in dynamic system monitoring," Mechanical Systems and Signal Processing, vol. 21, no. 2, pp. 706-723, 2007.

[34] S. H. Sim, B. F. Spencer Jr., and T. Nagayama, "Multimetric sensing for structural damage detection," Journal of Engineering Mechanics, vol. 137, no. 1, pp. 22-30, 2010.

[35] J. Kim, K. Kim, and H. Sohn, "Autonomous dynamic displacement estimation from data fusion of acceleration and intermittent displacement measurements," Mechanical Systems and Signal Processing, vol. 42, no. 1-2, pp. 194-205, 2014.

[36] S. H. Sung, J. W. Park, T. Nagayama, and H. J. Jung, "A multiscale sensing and diagnosis system combining accelerometers and gyroscopes for bridge health monitoring," Smart Materials and Structures, vol. 23, no. 1, Article ID 015005, 2014.

[37] G. W. Roberts, X. Meng, and A. H. Dodson, "Integrating a global positioning system and accelerometers to monitor the deflection of bridges," Journal of Surveying Engineering, vol. 130, no. 2, pp. 65-72, 2004.

[38] J.-W. Park, S.-H. Sim, and H.-J. Jung, "Displacement estimation using multimetric data fusion," IEEE/ASME Transactions on Mechatronics, vol. 18, no. 6, pp. 1675-1682, 2013.

[39] J.-W. Park, S.-H. Sim, and H.-J. Jung, "Wireless displacement sensing system for bridges using multi-sensor fusion," Smart Materials and Structures, vol. 23, no. 4, Article ID 045022, 2014.

[40] S. Cho, S.-H. Sim, J.-W. Park, and J. Lee, "Extension of indirect displacement estimation method using acceleration and strain to various types of beam structures," Smart Structures and Systems, vol. 14, no. 4, pp. 699-718, 2014.

[41] S. Cho, C.-B. Yun, and S.-H. Sim, "Displacement estimation of bridge structures using data fusion of acceleration and strain measurement incorporating finite element model," Smart Structures and Systems, vol. 15, no. 3-4, pp. 645-663, 2015.

[42] S. Sim, "Estimation of flexibility matrix of beam structures using multisensor fusion," Journal of Structural Integrity and Maintenance, vol. 1, no. 2, pp. 60-64, 2016.

[43] D. Gebre-Egziabher, R. C. Hayward, and J. D. Powell, "Design of multi-sensor attitude determination systems," IEEE Transactions on Aerospace and Electronic Systems, vol. 40, no. 2, pp. 627649, 2004.

[44] J. Schneider, C. Eling, L. Klingbeil, H. Kuhlmann, W. Forstner, and C. Stachniss, "Fast and effective online pose estimation and mapping for UAVs," in Proceedings of the IEEE International Conference on Robotics and Automation (ICRA '16), pp. 47844791, IEEE, Stockholm, Sweden, May 2016. 
[45] R. Sun, K. Han, J. Hu, Y. Wang, M. Hu, and W. Y. Ochieng, "Integrated solution for anomalous driving detection based on BeiDou/GPS/IMU measurements," Transportation Research Part C: Emerging Technologies, vol. 69, pp. 193-207, 2016.

[46] Y. Zhao, "Performance evaluation of Cubature Kalman filter in a GPS/IMU tightly-coupled navigation system," Signal Processing, vol. 119, pp. 67-79, 2016.

[47] Z. Chen, H. Zou, H. Jiang, Q. Zhu, Y. C. Soh, and L. Xie, "Fusion of WiFi, smartphone sensors and landmarks using the kalman filter for indoor localization," Sensors, vol. 15, no. 1, pp. 715-732, 2015.

[48] H.-Y. Chung, C.-C. Hou, and Y.-S. Chen, "Indoor intelligent mobile robot localization using fuzzy compensation and kalman filter to fuse the data of gyroscope and magnetometer," IEEE Transactions on Industrial Electronics, vol. 62, no. 10, pp. 6436-6447, 2015.

[49] http://www.sbg-systems.com. 


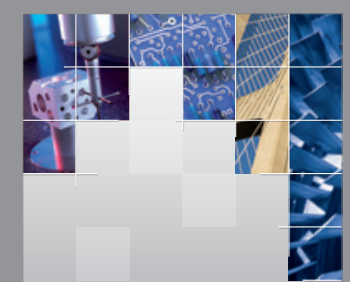

\section{Enfincering}
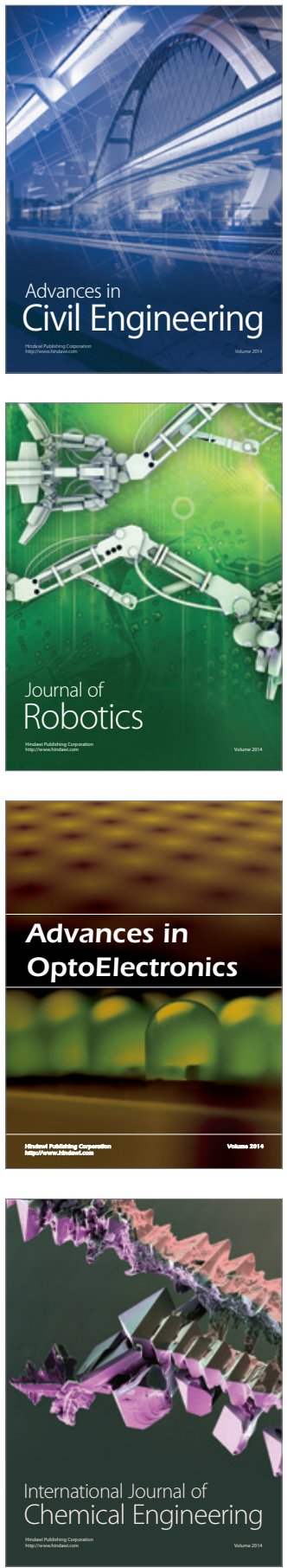

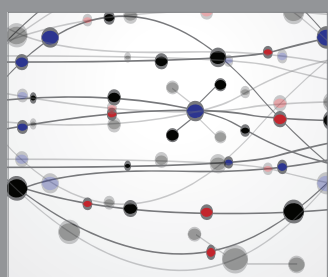

The Scientific World Journal

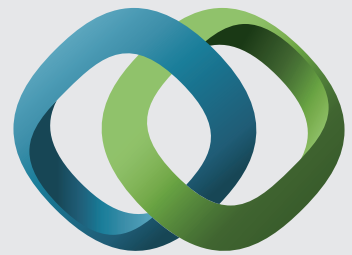

\section{Hindawi}

Submit your manuscripts at

http://www.hindawi.com
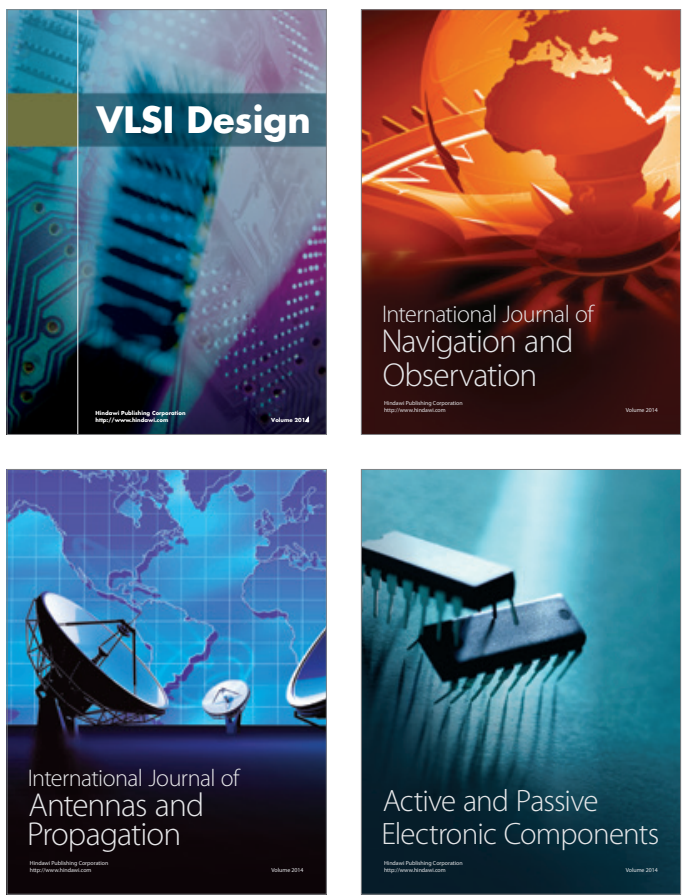
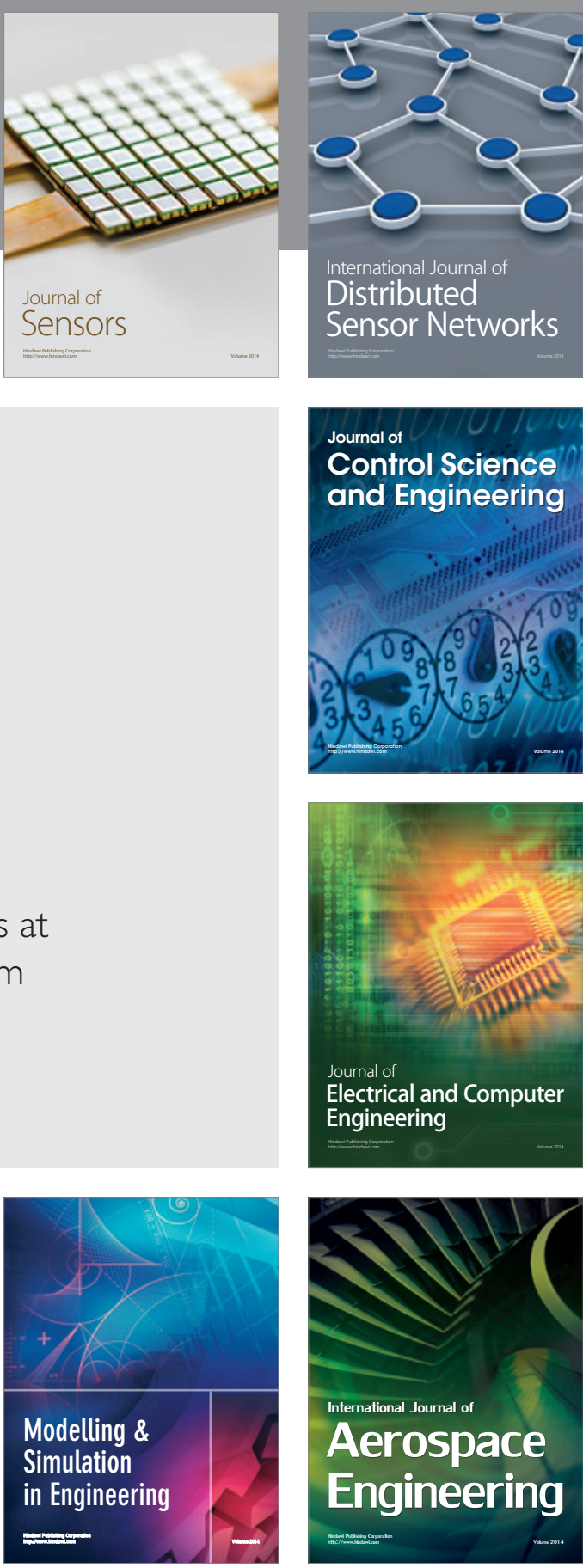

International Journal of

Distributed

Sensor Networks

Journal of

Control Science

and Engineering
